\title{
Procedure of selecting the priorities when constructing new most important facilities in the Russian energy sector in the context of materialization of strategic energy security threats
}

\author{
Viktor Rabchuk ${ }^{l}$, Sergey Senderov ${ }^{1,2, *}$, \\ ${ }^{1}$ Melentiev Energy Systems Institute of Siberian Branch of the Russian Academy of Sciences, 130 Lermontov str., Irkutsk, Russia \\ ${ }^{2}$ Federal State Budget Educational Institution of Higher Education «Irkutsk National Research Technical University», 83 Lermontov str., \\ Irkutsk, Russia
}

\begin{abstract}
The paper is devoted to finding ways to solve the problem of a scientifically based approach to the selection of priorities in the construction of new most important energy facilities in Russia for the future up to 15 years. The perspective scales of realization of strategic threats to the energy security of Russia are characterized. A list of subtasks that should be solved to form a methodology for solving the problem is shown.
\end{abstract}

\section{Introduction}

The paper concerns the ways to solve the problem of a scientifically-based selection of a list of most important facilities of the Russia's fuel and energy complex (FEC), which should be constructed as a priority in the medium term (up to 15 years). A most important facility from the standpoint of the development of the FEC in some perspective is the facility, the refusal to build which or its failure (in the case of construction) during operation can lead to an unacceptably significant reduction in domestic consumption of energy resources or in their export. The problem of choosing priorities, stated in the title of this paper, is becoming increasingly acute in the context of the active implementation of strategic threats to energy security (ES) in Russia. The current and expected scales of implementation of some of them are such that it is possible to talk about a possible process of containing economic growth from the energy sector in the medium term due to a significant deficit in the volume and duration of the supply of necessary types of energy $[1,2$, etc. $]$.

\section{Strategic threats to Russia's energy security today and in the short term}

In the list of ES strategic threats, it is worth highlighting the threat to a large extent determining the scale and dynamics of the development of other threats - the threat of a noticeable decrease in the investment opportunities of the national economy and energy sectors, in particular. Thus, the annual volume of investments during the period from 2011 to 2018 decreased: in the electric power industry - by $27 \%$ (from 893 to 648 billion rubles) $[3,4]$, in the gas industry - by $47 \%$ (from
647 to 340 billion rubles). Investments in the coal industry of Russia increased almost 1.5 times in 2018 compared with 2017 and amounted to 150 billion rubles [5], but earlier there was an active decline in their volumes, so in 2014 the decline in investment compared to 2012 was $37 \%$ and remained at this level until 2017 [6]. The situation is more stable in the sphere of oil production and transportation of oil and gas condensate, as well as in the field of oil refining (the total volume is about 1.4 trillion rubles [7]).

The uncertain situation with the investment opportunities of the country's energy industries is aggravated by the sharp deterioration in the capacity of large Russian companies (especially in the field of hydrocarbon production) to receive long-term low-cost borrowed funds. The increased negative consequences in the next 10-15 years will be caused by the introduction of the following strategic threats to the Russian ES:

- increase in the cost of production of Russian hydrocarbons and their transport to domestic consumers and for export (due to the depletion of the existing areas of their production and the need to enter new - more expensive - oil and gas production areas);

- the need for rapid growth in the coming years of the rate of replacement of physically and morally obsolete basic production assets (BPF) in the energy industries (in the last 10-15 years, the replacement rate of these BPF noticeably lagged behind the rate of aging, and their share today is clearly higher than the acceptable share);

- too high energy intensity of gross domestic product in Russia (it should be expected that in this perspective it will remain at the level of approximately one and a half times higher than in the Scandinavian countries and in Canada);

\footnotetext{
* Corresponding author: ssm@isem.irk.ru
} 
- deterioration of the situation for Russia, as an exporter of hydrocarbons, in world oil and gas markets (US shale hydrocarbons; expected largescale participation in the world markets of Iran and Iraq with their hydrocarbons; continuous growth in the share of unconventional energy in total energy resources in major countries - importers of oil and gas (China, Western European countries) and the general desire of these countries to reduce hydrocarbon purchases).

In addition to taking into account the expected degree of implementation of the strategic threats to the Russia's ES listed above, the solution of the identified problem ("choosing priorities") requires a comprehensive analysis and consideration of the following factors and requirements:

- the interconnectedness and interdependence of the work of all energy systems;

- the possibility of various large-scale emergencies in the basic sectors of the Russian FEC (gas and electric power industry) in the future;

- the expected dynamics of changes in the country's domestic energy needs in the same perspective;

- the need to diversify directions and forms (pipeline gas, liquefied natural gas) of Russian gas export and maintain the volumes of this export at the highest possible level;

- the need for a significant increase in the added value of produced Russian hydrocarbons (including due to the depth of oil refining, improving the quality of oil products and the intensive development of oil and gas chemistry).

"Selecting priorities" is part of the overall task of managing the development of the country's FEC for the analyzed perspective. To a certain extent, this problem was solved in the past $[8,9]$ and should be solved constantly in the future. Since the middle of the current decade, the situation with investments has worsened significantly, in particular in the energy sectors. Other strategic threats to energy security began to intensify (reinforcing the situation with a lack of investment). Under these conditions, the problem of "choosing priorities" has actually become the main part of the overall task of managing the development of the Russian FEC.

\section{Meaningful statement of the problem}

A meaningful statement of the problem of "choosing priorities" can be formulated as follows: it is necessary to create a list of new most important facilities from the standpoint of the Russia's FEC strategic development. Such facilities should be constructed on a priority basis until 2035, taking into account the dynamics of changes in the degree of implementation of strategic threats to the country's ES. Along with this, factors mentioned above and which may also affect the composition of the selected facilities of their specified list should be taken into account too.
The task of "choosing priorities" is complex, because when solving it, along with taking into account the strategic threats of the ES of Russia and the influence of various factors and requirements. The fact of the interconnectedness of the work of all energy sectors of the country within the framework of the functioning of the Russian FEC is taken into account too. The semantic content of the requirements for the methodology for solving this problem, which needs to be developed, speaks about the scale and difficulties of solving the problem of "choosing priorities".

Firstly, this methodology should determine the methods for obtaining an adequate assessment of the degree of realization of the strategic threat "lack of investment in the energy sectors of Russia" in the future. The same applies to the assessment in relation to other strategic threats of electronic security, the implementation of which enhances the negative effect of the lack of investment.

The second thing that is required from this methodology is clear instructions on the selection mechanism of those factors and requirements that must be taken into account when solving the problem of "choosing priorities" and on methods for taking these factors and requirements into account. Among the factors and requirements taken into account, at least, there should be those that have already been indicated above (the interconnectedness of functioning of the energy industries, the expected dynamics of changes in the country's domestic energy needs, the need to diversify the directions and forms of export of Russian hydrocarbons).

The methodology should determine the principles of concretizing the concept of "unacceptably significant reduction in domestic energy resources consumption and their export", which was mentioned at the beginning of the paper when determining the meaning of the term "most important new FEC facility" for the certain perspective. Concretizing in this case will relate to quantitative indicators of the reduction in domestic consumption of fuel and energy resources and their exports in case of refuse to build this new FEC facility (or failure of an already constructed facility during its operation). The developed methodology should determine the general procedure for solving the entire considered full-scale task of "choosing priorities".

The task of "choosing priorities" is solved mainly through the use of formalized approaches with the use of expert analysis methods at certain stages. The use of formalized approaches requires a prepared mathematical formulation of both the entire problem to be solved and its individual stages, indicating the criteria and limitations used. As mentioned above, a reasonable choice of these criteria and limitations is the main requirement for the developed methodology for solving the problem.

\section{The main subtasks of the general task of "choosing priorities"}


Already today, in parallel with the development of the methodology mentioned above, we can begin to prepare for the fulfillment of the sub-tasks of the general task of "choosing priorities", which will have to be fulfilled. These subtasks include:

1. Formation (with appropriate justification) of the list of Russia's ES strategic threats, which will be implemented in the period up to 2035 and the scale of implementation of which can significantly affect the solution of the issues of creating new most important FEC facilities in the analyzed period.

2. The solution of the methodological issues of assessing the scope, nature of the transformation and the degree of implementation of Russia's ES strategic threats to the period up to 2035, including the development of new and adaptation of existing models to obtain this assessment. Substantiation of the procedure for taking this assessment into account in the "choosing priority" task when deciding on the creation of new most important FEC objects.

3. Assessment of the scales and nature of the transformation, until 2035, of the selected Russia's ES strategic threats using new developed and existing adapted models with an assessment of the degree to which each threat is realized in the analyzed prospect.

4. The solution of methodological issues of accounting for various factors and requirements (including the order of their choice) to solve the problem of "choosing priorities".

5. Adaptation of existing models to solve the problem of managing the Russian FEC development in order to obtain a formalized solution to the considered problem of "choosing priorities".

6. The information content of the adapted models in accordance with the essence of the substantive and mathematical formulations of the problem of "choosing priorities" and using the results of assessing the expected degree of implementation of Russia's ES strategic threats up to 2035. Here we should also select the necessary information on various factors and requirements that can impact on the "choice of priorities".

7. The use of adapted models after their information content to obtain a basic solution to the task of "choosing priorities".

8. Assessment of the possibilities of referring to the list of critical facilities (CF), facilities that in the formalized solution of the "choosing setting" task were included in the list of priority constructed new most important facilities of the FEC for the future until 2035 (done by modeling the functioning of these facilities in the context of large-scale emergency situations in the fuel and energy sectors). A number of issues related to the adaptation of the currently used approaches, methods and models with the analysis of the significance of a particular CF should also be preliminarily resolved.

9. Correction of the formalized solution of the task of "choosing priorities" using the results of expert evaluation. At the same time, the impact on the resulting solution of factors that are almost impossible to take into account by formalized methods (the uncertainty factor, a number of subjective factors, the feasibility of the solution, etc.) is evaluated. The final solution to the task of "choosing priorities" when considering the creation of new most important facilities of the Russia's FEC until 2035.

\section{Conclusion}

Until now, when solving the tasks of managing the development of the Russia's FEC, the effect of the strategic threats to the country's ES was not actually taken into account. Perhaps this was permissible in the first decade of the 21 st century, when the degree of realization of these threats did not reach nowadays level. It is extremely important now to take into account the conditions for the implementation of factors such as: lack of investment, growth in unit costs for the production and transportation of hydrocarbons throughout Russia, a noticeable lag in the rate of replacement of fixed assets in the energy sector from the rate of their physical and moral aging, and others. Failure to take them into account when solving the problems of managing the development of the FEC leads, as a rule, to obtaining too optimistic solutions (especially regarding the expected production capabilities of the energy sectors). The result is a situation of unacceptably high risk of disruption of the process of reliable fuel and energy supply to consumers in emergency situations of various kinds and the formation of barriers for sustainable energy development in the future. The conventionally old approach to solving the problems of managing the development of the fuel and energy complex does not make sense not only for the future of 30-40 years (due to too much uncertainty in the future), but also for the medium term, mainly due to the lack of consideration of the effect of the ES strategic threats. The application of the approach proposed in this paper will increase the degree of confidence in the results of solving the problems of managing the Russia's FEC development and create a methodological basis for minimizing the negative consequences for consumers of fuel and energy resources within the country in the context of the implementation of ES strategic threats in the future.

The work was carried out within the framework of a scientific project III.17.5.1 of program of fundamental research of the SB RAS, reg. number AAAA-A17-117030310451-0 and RFBR research project №18-58-06001, reg. number AAAA-A18118050490009-5.

\section{References}

1. Rabchuk V.I., Senderov S.M., Izv. RAN. Energetika [Proc. of the Russ. Acad. of Sciences. Pow. Engin.], 1, 50-58 (2019).

2. V. Rabchuk, S. Senderov Proceedings International Conference Green Energy and Smart Grids (2018). DOI: $10.1051 / \mathrm{e} 3$ sconf/20186902011. 
3. S. Senderov, V. Rabchuk, N. Pyatkova, S. Vorobev Ensuring Russia's Energy Security: A Choice of Priorities (Nauka, Novosibirsk, 2017).

4. https://www.acra-ratings.ru/research/691.

5. https://tass.ru/ekonomika/6092746.

6. Volokitina V.M., Gedich T.G., Economicheskii analiz: teoria I practika [Economic analysis: theory and practice], 16 (7), 1260-1268, (2017).

7. https://rns.online/energy/Novak-nazval-ozhidaemiiobem-investitsii-v-TEK-Rossii-v-2019-godu--201904-05/.

8. Voropai N.I., Kler A.M. \& oth. System studies in the energy sector: methodology and results (ERI RAS, Moscow, 2018).

9. Fillippov S.P., Dilman M.D. System studies in the energy sector: methodology and results (ERI RAS, Moscow, 2018). 